\title{
Correction to: Immunometabolic Pathways and Its Therapeutic Implication in Autoimmune Diseases
}

\author{
Tingting Wang ${ }^{1,2,4} \cdot$ Yuhao Jiao ${ }^{2,3} \cdot$ Xuan Zhang $^{2,3,4}$
}

Published online: 27 November 2020

○) Springer Science+Business Media, LLC, part of Springer Nature 2020

\section{Correction to: Clinic Rev Allerg Immunol (2020) https://doi.org/10.1007/s12016-020-08821-6}

In PDF of the recently published article "Immunometabolic Pathways and Its Therapeutic Implication in Autoimmune Diseases," the article note "Tingting Wang and Yuhao Jiao have contributed equally" is missing. This should be added.

The original article has been corrected.

The original article can be found online at https://doi.org/10.1007/ s12016-020-08821-6.

\section{Xuan Zhang}

zxpumch2003@sina.com

1 Department of Medical Research Center, Peking Union Medical College Hospital, Peking Union Medical College and Chinese Academy of Medical Sciences, Beijing 100730, China

2 Clinical Immunology Centre, Medical Epigenetics Research Centre, State Key Laboratory of Difficult and Severe and Rare Diseases, Peking Union Medical College Hospital, Chinese Academy of Medical Sciences and Peking Union Medical College, Beijing 100730, China

3 Department of Rheumatology and Clinical Immunology, Peking Union Medical College Hospital, The Ministry of Education Key Laboratory, Chinese Academy of Medical Sciences and Peking Union Medical College, Beijing 100730, China

4 State Key Laboratory of Difficult, Severe and Rare Diseases, Peking Union Medical College Hospital, Peking Union Medical College and Chinese Academy of Medical Sciences, Beijing 100730, China 